\title{
Intracranial aneurysm surgery and its future
}

\author{
R S Maurice-Williams FRCP FRCS J Lafuente FRCS
}

The history of intracranial surgery for aneurysms is not a long one. The first direct operation on an intracranial aneurysm was performed by Norman Dott, who wrapped a ruptured aneurysm in $1933,{ }^{1}$ and the first obliterative clipping of an aneurysm was performed by Walter Dandy in $1938 .^{2}$ The results of surgery improved dramatically when the operating microscope was introduced in the $1960 \mathrm{~s}^{3}$ and a subsequent improvement followed the use of the calcium antagonist nimodipine and the maintenance of a high fluid intake to lessen the risk of delayed cerebral ischaemia. ${ }^{4}$

For many years clipping of a ruptured aneurysm was regarded as the definitive mode of treatment, but the development of the GDC coil in 1990 allowed an alternative approach that avoided the hazards of open surgery. In the latter half of the 1990s, as experience of endovascular techniques spread, this form of treatment began to displace open surgery and the International Subarachnoid Aneurysm Trial (ISAT) was set up to compare the efficacy of the two forms of treatment.

Early in 2002 the trial was interrupted by the steering committee when an intermediate analysis of the results revealed that, in the short term at any rate, the results of endovascular coiling were superior to those of clipping, in that the chances of survival free of disability at one year were significantly better for coiling. Furthermore, the risk of rebleeding from a successfully coiled aneurysm at one year was only $0.16 \% .^{5}$

Although this still leaves open the long-term risks of aneurysm re-formation and rebleeding after coiling (a risk which is negligible after successful clipping), we believe that the results of ISAT must spell the end of aneurysm surgery-though no doubt there will be a transitional period of some years before surgery finally disappears. As things stand, it is difficult to advise surgery in those cases where coiling is practicable, and publication of the ISAT probably led to an immediate shift towards coiling in most units in the UK.

The current margin in favour of endovascular treatment is likely to increase in the future, for the following reasons. Endovascular as opposed to surgical treatment is an evolving technique. Not only is the microtechnology constantly improving but also more and more neuroradiologists are acquiring and honing their skills in this field. As this happens, increasing proportions of aneurysms are proving amenable to endovascular treatment. By contrast, the technology of surgery has been static for many years and, after the quantum leap in results provided by the introduction of the operating microscope and associated microinstrumentation, it is difficult to see how the techniques of open surgery could be further improved. Furthermore, as the use of endovascular techniques extends, so the surgical expertise will be lost. Already the pool of very experienced surgeons is draining away as a result of death and retirement, and as these surgeons disappear the advantages of endovascular treatment will become greater still. The number of cases unsuitable for endovascular treatment will shrink to the point where there will be a residue of difficult and complex cases for which only surgery is available. However, it is by no means certain that surgery would be justified in such cases. These are likely to be patients with, for example, giant and complex aneurysms in inaccessible situations where even at present surgical as opposed to conservative treatment may be difficult to justify. This will be still more true when the surgical skills honed upon hundreds of more straightforward cases have been lost.

For these reasons, any review of aneurysm surgery must be largely of historical interest. However, we think it of value to touch on some topics that have concerned the senior author in the course of 30 years of aneurysm surgery during which time he has treated almost a thousand cases. Over this period he had prepared prospective datasheets for every patient treated under his care. These datasheets have provided much information on the clinical course of the patients treated, the technical difficulties encountered at surgery and the postoperative problems that arose.

\section{NATURAL HISTORY OF RUPTURED ANEURYSM}

Much of what has been written about the natural history of subarachnoid haemorrhage has been based on findings of the Multi-centre Cooperative Trial which began in the 1960s. Subsequently it has become apparent that investigators at that time took an over-simple view of the sequence of events that may follow rupture of an aneurysm. They were largely concerned with the occurrence of rebleeding and almost certainly overestimated the incidence of this event. Their published charts showed risk of bleeding rising to a peak between days five and nine. ${ }^{6}$ What they did not realize 
was that not all episodes of acute deterioration are caused by rebleeding and that non-haemorrhagic deterioration (NHD) is almost twice as common as rebleeding, though less serious, carrying a mortality of about $20-25 \%$ as opposed to $60-70 \% .{ }^{7}$ NHD is often clinically indistinguishable from a rebleed, and the principal causes are cerebral vasospasm and raised intracranial pressure consequent upon obstruction of the cerebrospinal fluid pathways or local brain swelling.

A paper published in $1982^{7}$ showed that episodes of rebleeding as confirmed by CT scanning, lumbar puncture or autopsy had a flat distribution in time, whereas episodes of clinical worsening where rebleeding could not be confirmed reached a peak between days five and nine corresponding to the period of maximal reactive vasospasm (which tends to develop three days or so after rupture of an aneurysm). It was clear that the Cooperative Study had confused NHD with rebleeding and had considerably overestimated the occurrence of the latter - a fact with clear implications for the timing of surgery. More recently, it has been asserted that there is a peak of very early rebleeds (within the first 24 hours) which justifies a policy of ultra-early surgery; ${ }^{8}$ however, despite careful monitoring of the patients under our care, we have found no evidence for such very early rebleeding - indeed, a second haemorrhage would be hard to diagnose with certainty so soon after the presenting one.

Another feature of the natural history that has been overlooked is the fact that patients who survive the initial haemorrhage in good condition have a remarkably good long-term prognosis even with conservative management. It is possible that such patients have a low incidence of rebleeding; furthermore, their low risk of NHD probably reflects the lesser blood loading of the cerebrospinal fluid after a mild first bleed. This benign natural history for goodcondition patients was pointed out both in the Cooperative Study ${ }^{9}$ and in a study by Alvord ${ }^{10}$ which related the chances of a patient surviving for two years against the patient's clinical grade and the interval after the presenting haemorrhage. Thus, for a patient in grade 1 who survives the first four days after the presenting bleed there is a $90 \%$ chance of surviving for two years even without active treatment. Again, these figures have major implications for active treatment. Is it unduly cynical to suppose that Alvord's study was widely ignored because it did not fit in with the policy of early and aggressive surgical treatment so popular over the past 10-15 years, especially in countries with a superabundance of neurosurgeons?

\section{SURGICAL AND ANAESTHETIC TECHNIQUES}

The techniques used for aneurysm surgery have for long been standardized. 95\% of aneurysms are accessible via a small frontotemporal craniotomy centred over the pterion. Only occasional cases, principally aneurysms of the distal anterior cerebral artery and the lower vertebrobasilar trunk, require different surgical approaches. The risk of intraoperative rupture of the aneurysm, which occurs in $5-10 \%$ of cases, can be minimized by induction of hypotension. This seems a safe measure provided that the anaesthetist ensures maintenance of a high blood volume. Some surgeons have applied temporary clips to the main artery proximal to the aneurysm but in our experience this technique is associated with a high incidence of ischaemic cerebral damage. Temporary clipping is certainly not tolerated if the blood pressure is lowered at the same time.

\section{TIMING OF SURGERY}

During the past twenty years a major controversy concerned the timing of surgery. In the 1970s the axiom was that surgery should be delayed for ten or more days from the presenting bleed even in patients who were in a good condition. ${ }^{11}$ Such delay allowed the initial brain swelling and cerebrovascular instability to subside. Delayed surgery, it was argued, was technically easier than surgery performed within the first few days and gave much better results. Since that time there has been an increasing tendency towards early surgery even in poor-condition patients, with the intention of preventing rebleeding and permitting the use of postoperative hypertension and hypervolaemia to lower the incidence of ischaemic brain damage. ${ }^{12}$ The senior author is one of that minority who continued with the previous policy of delayed surgery. He has found that, although $4-5 \%$ of patients in grades $1-3$ will die from rebleeding while waiting, in some of these cases the fatal rebleed occurs so early that it would not have been prevented by early surgery. This has left about 3\% of patients whose lives might have been saved by early surgery ${ }^{13}$ - which is approximately the proportion of patients who would have been lost as a result of technical difficulties had we changed to early surgery. On existing evidence, the timing of surgery has little if any effect on the overall management results. ${ }^{14}$ Soon this will become a forgotten and irrelevant controversy. With endovascular treatment there seems no argument for delay, and this will have profound implications for the lives of neuroradiologists since patients will need to be treated within a few hours after arrival at the specialized centre.

\section{INTERPRETATION OF SURGICAL RESULTS}

The results of surgery in different series have been difficult to compare because numerous factors affect outcome. Amongst these are the condition of the patient at the time of surgery and the interval from the presenting bleed. There is also the question as to whether the focus is purely on surgical results or on the overall results of all patients whether or not surgery is performed. The interval from 
treatment is also important since many patients die or change in condition after the conventional period of thirty days from surgery; there is much to be said for assessing outcome at one year at least. Preadmission policies also influence the outcome. ${ }^{15}$ Any selectivity or delay in patient transfer can skew the treated population by preventing patients who would otherwise do badly from reaching neurosurgical care. Even with an open-door policy for admissions - taking all patients irrespective of their condition as soon as possible after referral — we have found that only $55 \%$ of patients reach neurosurgical care within 24 hours of the ictus and only $75 \%$ within 72 hours. These figures have remained constant over many years. Furthermore, only about $45 \%$ of patients who arrive within 24 hours are in good condition (grades 1 and 2) whereas $85 \%$ of patients who arrive for neurosurgical care later than seven days are in good condition. This reflects the fact that the delayed diagnosis is more likely with a minor subarachnoid haemorrhage than with one that has left the patient neurologically devastated.

The assessment of results can easily reflect bias, especially if the assessment is made by the treating surgeon. There are also further problems. Most surgical series have assessed outcome according to the Glasgow outcome scale, ${ }^{16}$ and by convention the result has been recorded as good if the patient is left in one of the two highest categories of the scale. However, a patient may be in the highest category but still have residual neurological and psychological dysfunction, whilst in the next category down ('moderate disability') the patient may have major neurological disability despite qualifying for this category in being independent as regards the activities of daily life.

\section{THE EFFECT OF EXPERIENCE}

Aneurysm surgery is delicate, performed under the operating microscope deep under the base of the brain. It would be surprising if surgical results were not affected by experience and technical skills - a point which will clearly be important in the future when few if any experienced aneurysm surgeons will be available. A clear learning curve for the senior author was evident over the period of the first 400 ruptured aneurysms he had treated. ${ }^{17}$ During successive 100-patient cohorts with the same open-door admission policy, the overall management mortality fell steadily from $38 \%$ to $24 \%$ while one-year surgical mortality fell from $19 \%$ to 3\%. During this time the proportion of patients operated on rose from $62 \%$ to $72 \%$.

\section{THE CAUSES OF POOR SURGICAL RESULTS}

In the same series of patients we looked at the causes of a regards important residual neurological disability. ${ }^{17}$ Of a total of 24 deaths in the first year after surgery only 2 were the result of preoperative events (overwhelming brain damage from the primary bleed) and only 2 were the result of postoperative complications (one subdural empyema and one case of progressive cerebral vasospasm). The remaining 20 deaths were due to technical mishaps or failures - events that declined in frequency with increasing experience from $15 \%$ to $1 \%$ over successive cohorts of 100 patients.

In the group of surgical mishaps were included such events as inadvertent occlusion of a normal artery by the clip, and failure to control intraoperative haemorrhage from the aneurysm. In the operative-failure category were patients who re-bled after wrapping of an aneurysmalways a second-best option, since clipping excludes the aneurysm from the circulation.

By contrast, in 20 patients who survived but were still disabled one year after surgery, the neurosurgical damage appeared to have been caused by preoperative events in 13 and by postoperative events in 3 (all cases of cerebral vasospasm), leaving only 4 in whom events during the surgery itself seemed to have caused the disability.

\section{SURGERY TOMORROW?}

By the time of the coiling era, the effect of surgical experience had levelled off for the senior author but an analysis of his results during this stable surgical period shows why coiling is certain to replace surgery. At first sight the operative results are satisfactory. ${ }^{18}$ Of 245 patients with ruptured aneurysms treated over a five-year period until 1999, 190 (78\%) underwent surgical treatment. In this latter group the one-year mortality was $2.6 \%$, and $83.7 \%$ were in the highest category of the Glasgow outcome scale. The overall management results were of course less good, with a one-year mortality of $17.1 \%$ and only $67.4 \%$ in the best outcome-scale category, reflecting the fact that many of the non-operated cases arrived in poor condition or deteriorated before surgery could be done.

However, 29.5\% of the 190 operated patients had experienced complications of some sort, either cranial or systemic, and $20 \%$ of the operated patients required subsidiary operations, these being mainly for hydrocephalus, cerebral oedema or intracranial collections of blood or fluid. In all probability, these complications and further procedures would have been largely avoided if coiling had been in use - a conclusion strong enough in itself to tip the balance in the direction of endovascular treatment. The results of the ISAT study further strengthens the case for coiling. 


\section{REFERENCES}

1 Dott NM. Intracranial aneurysms cerebral arterio-radiography and surgical treatment. Edinb Med J 1933;40:219-34

2 Dandy WE. Intracranial aneurysm of the internal carotid artery cured by operation. Ann Surg 1938;107:654-9

3 Krayenbuhl HA, Yasargil MG, Flamm ES, et al. Microsurgical treatment of intracranial saccular aneurysms. J Neurosurg 1972;37:678-86

4 Pickard JD, Murray GD, Illingworth R, et al. Effect of oral nimodipine on cerebral infarction and outcome after subarachnoid haemorrhage. British Nimodipine Trial. BMJ 1989;298:636-42

5 Intracranial Subarachnoid Aneurysm Trial (ISAT) Collaborative Group Intracranial Subarachnoid Aneurysm Trial (ISAT) of neurosurgical clipping versus endovascular coiling in 2143 patients with ruptured intracranial aneurysms - a randomised trial. Lancet 2002;360:1267-74

6 Locksley MB. Natural history of subarachnoid hemorrhages, intracranial aneurysms and arteriovenous malformations. J Neurosurg $1966 ; 25: 321-68$

7 Maurice-Williams RS. Ruptured intracranial aneurysms: has the evidence of early rebleeding been over-estimated? J Neurol Neurosurg Psychiatry 1982;45:774-9

8 Kassell NF, Torner JC. Aneurysmal rebleeding: a preliminary report from the Cooperative Aneurysm Study. Neurosurgery 1983;13:479-81

9 Nishioka H. Evaluation of the conservative management of ruptured intracranial aneurysms. J Neurosurg 1966;25:574-92
10 Alvord EC, Loeser JD, Bisley WL, et al. Subarachnoid hemorrhage due to ruptured aneurysms: a simple method of estimating prognosis. Arch Neurol 1922;27:273-91

11 Adams CB, Loach AB, O'Laoire SA. Intracranial aneurysms: analysis of results of microneurosurgery. BMJ 1976;ii:607-9

12 Spetzer V, Gilsbach JM. Results of early aneurysm surgery in poorgrade patients. Neurol Res 1994;16:27-30

13 Maurice-Williams RS, Wadley JP. Delayed surgery for ruptured intracranial aneurysms: a reappraisal. Br J Neurosurg 1997;11:104-9

14 Kassell NF, Torner JC, Haley EC, et al. The International Cooperative Study on the timing of Aneurysm Surgery. Part 1: overall management results. J Neurosurg 1990;73:18-36

15 Maurice-Williams RS, Marsh H. Ruptured intracranial aneurysms: the overall effect of treatment and the influence of patient selection and data presentation on the reported outcome. I Neurol Neurosurg Psychiatry 1985;48:1208-12

16 Jennett B, Bond M. Assessment of outcome after severe brain damage. Lancet $1975 ; \mathbf{i}: 480-4$

17 Maurice-Williams RS, Kitchen ND. Ruptured intracranial aneurysms - learning from experience. Br J Neurosurg 1994;8:519-27

18 Lafuente J, Maurice-Williams RS. Ruptured intracranial aneurysms: the outcome of surgical treatment in experienced hands in the period prior to the advent of endovascular coiling. J Neurol Neurosurg Psychiatry (in press) 